\title{
The time course changes in expression of aquaporin 4 and aquaporin 1 following global cerebral ischemic edema in rat
}

\author{
Gökhan Akdemir, Figen Kaymaz'1, Yasemin Gursoy-Özdemir, Nejat Akalan, Ekin Su Akdemir² \\ Institute of Health Sciences, Hacettepe University, ${ }^{1}$ Department of Histology and Embryology, Faculty of Medicine, Hacettepe University, Altindağ 06100, Ankara, \\ ${ }^{2}$ Department of Molecular Biology and Genetic, Boğazici University, Bebek 34342, Istanbul, Turkey \\ E-mail: *Gökhan Akdemir - gokhanakdemir@yahoo.com; Figen Kaymaz -ffkaymaz@yahoo.com;Yasemin Gursoy-Özdemir - yozdemir@hacettepe.edu.tr; \\ Nejat Akalan -nejata@tr.net; Ekin Su Akdemir - ekinsuakdemir@gmail.com \\ *Corresponding author
}

Received: 06 October $15 \quad$ Accepted: 10 November $15 \quad$ Published: 06 January 16

\begin{abstract}
Background: The aim of this global cerebral ischemia study was to study the changes in expression levels of aquaporin 4 (AQP4) and AQP1 over time.

Methods: Sprague-Dawley type male rats were divided into six groups. Sham group and ischemia/reperfusion were performed on five other groups using the four-vessel occlusion model. Reperfusion was done $30 \mathrm{~min}$ after the occlusion, and each group was tested at 1, 6, 12, 24, and $48 \mathrm{~h}$ for brain wet-dry weight ratio and AQP4 and AQP1 expression levels using immunohistochemistry. To prove ischemia development exists in both hippocampal neurons and epithelia of choroid plexus, hematoxylin, and eosin and neuronal marker (NeuN) immune-staining have been applied to the sham experimental group at $48 \mathrm{~h}$. AQP4 expression levels are also measured with western blotting.

Results: After ischemia/reperfusion it is observed that the decrease in brain water content between 12 and $24 \mathrm{~h}$ was statistically significant $(P<0.01)$. In parallel and based on immunohistochemical staining, AQP4 expression levels did not exhibit any statistically significant change. AQP4 levels did not show any statistically significant change in western blotting. AQP1 expression in choroid plexus epithelial cells decreased at the 12 and $24 \mathrm{~h}$ but increased in $48 \mathrm{~h}(P<0.05)$.

Conclusions: Lack of change in AQP4 expression levels is thought as its dual role in formation and elimination of ischemic brain edema. Decrease in AQP1 expression levels in $24 \mathrm{~h}$ can be explained with necrosis in choroid plexus after ischemia and the increase in $48 \mathrm{~h}$ mark can be related to recovery in choroid plexus.
\end{abstract}

Key Words: Aquaporin 1, aquaporin 4, brain edema, global cerebral ischemia/reperfusion

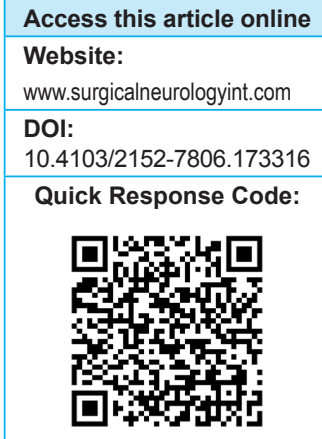

\section{INTRODUCTION}

Stroke is a major cause of morbidity and mortality in most developed countries. ${ }^{[4]}$ Stroke leads to global ischemia, which is caused by cardiac arrest, near drowning, and hypotension. Cerebral edema occurs frequently following ischemic stroke. Brain edema is an abnormal accumulation of fluid within brain tissue, which can be caused by a range of medical conditions,
This is an open access article distributed under the terms of the Creative Commons Attribution-NonCommercial-ShareAlike 3.0 License, which allows others to remix, tweak, and build upon the work non-commercially, as long as the author is credited and the new creations are licensed under the identical terms.

For reprints contact: reprints@medknow.com

How to cite this article: Akdemir G, Kaymaz F, Gursoy-Özdemir Y,Akalan N, Akdemir ES. The time course changes in expression of aquaporin 4 and aquaporin I following global cerebral ischemic edema in rat. Surg Neurol Int 2016;7:4. http://surgicalneurologyint.com/The-time-course-changes-in-expression-ofaquaporin-4-and-aquaporin-I-following-global-cerebral-ischemic-edema-in-rat/ 
including stroke, traumatic brain injury, brain tumors, and brain abscess. ${ }^{[8]}$ Brain edema increases intracranial pressure (ICP), potentially leading to brain ischemia, shifting, herniation and death. It is classified mainly into cytotoxic and vasogenic edema. ${ }^{[7]}$ Cytotoxic edema refers to primarily astrocytes cells swelling due to the failure of cellular energy metabolism. Vasogenic edema is produced by the increase of water, protein, and salt volume into the intercellular space of the brain tissue due to the breakthrough of blood-brain barrier (BBB) permeability. ${ }^{[16]}$

Aquaporins (AQPs) are a family of molecular water channel proteins that facilitate water transport across the plasma membranes of cells in response to osmotic gradients. ${ }^{[1,18]}$ Three AQPs have been identified, AQPl, AQP4, and AQP9 in the rodent brain. ${ }^{[2]}$ AQP4 is the principle bidirectional water transporting channel expressed in astrocytes throughout the central nervous system (CNS), mainly at astrocyte endfeet at the BBB, ependymal cells of the ventricles. ${ }^{[9,12]}$ Recent studies hypothesized that in glymphatic where perivascular astrocytic end feet that immediately bound at perivascular cerebrospinal fluid (CSF) influx and interstitial fluid efflux, AQP4 has a critical role CSF transportation. ${ }^{[17]} \mathrm{AQPl}$ is primarily expressed within epithelial cells of the choroid plexus in the ventricles and also has been proposed to have a role in production of CSF.[1] AQP9 is an aquaglyceroporin in astrocytes of the white matter may contribute to the regulation of brain energy metabolism. ${ }^{[3]}$

AQP4 and AQP1 appear to play critical roles in cytotoxic edema formation and as well as resolution in vasogenic edema. In this study, we explored that the time course changes of AQP4 and AQPl expressions in a rat model of four-vessel ischemia-reperfusion.

\section{MATERIALS AND METHODS}

\section{Animals}

Sprague-Dawley type male (weight 180-250 g) rats were used in this study. They were housed under diurnal lightning conditions and fasted overnight but allowed free access to water after the surgical procedure. Animal housing, care, and application of experimental procedures were all carried out in accordance with the institutional guidelines and approved by the Hacettepe University Animal Experiments Local Ethics Committee.

Four-vessel occlusion model

Rats were randomly divided into six groups. One group was the sham group, and ischemia/reperfusion was performed on the other five groups on different hours (l h, $6 \mathrm{~h}, 12 \mathrm{~h}, 24 \mathrm{~h}$, and $48 \mathrm{~h})$ using the four-vessel occlusion model. Rats were anesthetized with intraperitoneal administration of $90 \mathrm{mg} / \mathrm{kg}$ ketamine $\left(K_{e} a l a r{ }^{\circledR}\right.$ Ketamine Hydrochloride Injection, USP,
Parke-Davis, Morris Plains, NJ 079950 ABD), and $10 \mathrm{mg} / \mathrm{kg}$ xylasine $\left(\right.$ Rompun $^{\circledR}$, xylazin hydrochlorid $2 \%$, Bayer, Mefar İlaç San. A.Ş., Istanbul, Turkey).

After confirming deep anesthesia, a midline incision was made with Carl-Zeiss OPMI-l-FC microscope at $\times 1$ to $\times 1.6$ magnifications (Carl Zeiss, GmbH, Germany) in the dorsal neck and the cervical muscles were divided down to the atlanto-occipital junction. To produce experimental global cerebral ischemia, the alar foramina of the atlas were identified, and a bipolar bayonet (Codman Bipolar Johnson and Johnson Co., ABD) was passed in each foramina to electro-coagulate the underlying vertebral artery, and then the muscles and fascia were sutured closed in layers, as a previously reported rat model. ${ }^{[14]}$ After $24 \mathrm{~h}$, the animals were placed in the supine position, a mid-cervical skin incision was made in the anterior neck and thymus, and sternocleidomastoid muscles were retracted to expose the common carotid arteries. Both carotid arteries were clipped at nearly the same time using Aesculap-Yasargil titanium temporary miniclips (Aesculap AG, Tutlingen, Germany). After $30 \mathrm{~min}$, both clips were removed by clip applicator and skin was sutured. Body temperature was monitored by rectal probe and maintained at $36.5-37.5^{\circ} \mathrm{C}$ using a heat pad and overhead lamp. Rats were anesthetized overdose with pentobarbital 100 mg/kg (ABBOTT Laboratuarları A.Ş. İstanbul, Turkey) and brain was removed and divided two hemispheres. One of the hemispheres is weighted for brain wet-dry weight ratio, and the other hemisphere is used for hematoxylin and eosin stain ( $\mathrm{H}$ and $\mathrm{E}$ ), immunohistochemistry and western blotting.

\section{Brain water content measurement}

Brains were removed and divided into an ipsilateral and contralateral hemisphere. The ipsilateral hemisphere tissue was placed on a piece of aluminum foil and weighted immediately (wet weight) and dried in vacuum oven at $70^{\circ} \mathrm{C}$ for $24 \mathrm{~h}$. The dried brains were reweighted, and brain water content was calculated as (wet weight - dry weight)/(wet weight $\times 100)$.

Hematoxylin and eosin staining and immunohistochemistry

Brains were removed control group and at $1 \mathrm{~h}, 6 \mathrm{~h}, 12 \mathrm{~h}$, $24 \mathrm{~h}$, and $48 \mathrm{~h}$ after four-vessel occlusion. Contralateral hemispheres are waited in fluid nitrogen $-196^{\circ} \mathrm{C}$ for 3 days. Frozen sections of $7 \mu \mathrm{m}$ thickness were cut in the coronal plane (beginning at the bregma and extending caudally) on a Leica (Jung Frigocut 2800E) cryostat and air dried. After the sections were $\mathrm{H}$ and $\mathrm{E}$ and examine Leica DM 6000B microscope (Leica Microsystems, Wetzlar, Germany).

The sections were immune-stained at the room temperature for $1 \mathrm{~h}$ with rabbit anti AQP4 and AQPl (1/100 Chemicon AB3594 USA) and mouse anti-NeuN (1:200; Millipore Chemicon USA) followed by the 
appropriate fluorescent secondary antibody (1:200; Invitrogen-Life Technologies, Carlsbad, CA, USA) or biotinylated secondary antibody (1:500; Vector Laboratories, Burlingame, CA, USA). Tissue sections were examined with a Leica DM 6000B microscope (Leica Microsystems, Wetzlar, Germany).

\section{Immunohistochemistry analysis}

Immunoreactivity of the $\mathrm{AQP} 4$ and $\mathrm{AQPl}$ was quantified with fluorescence intensity using macnification vl.6.2 analysis software (Orbicule Inc., Leuven, Belgium) with an iMac computer $(3.06 \mathrm{GHz}$ Intel Core 2 Duo Apple Co., USA). The level of fluorescence was quantified in the region of interests (ROIs) analysis. AQP4 immunoreactivity was quantified at brain contralateral hemisphere; AQPl immunoreactivity was quantified in choroid plexus of the third ventricle.

\section{Western blotting of aquaporin 4}

Expression of AQP4 was quantified with western blotting. It is performed with the anti-AQP4 antibody (rat, polyclonal, Chemicon, USA). An equal amount of proteins per condition are separated on a $10 \%$ Novex Bis-Tris (Invitrogen, USA) gel and electrotransferred onto polyvinylidene fluoride membrane (Invitrogen, USA). Immunoreactive bands were visualized with Kodak Image Station $2000 \mathrm{MM}$ and intensity levels are measured with NIH image program.

\section{Statistical analysis}

Immunoreactivity ROI and water content were expressed as mean \pm standard error (generally 4-6 mice per each group). A significant difference was defined as $P<0.05$. Group comparisons were made with Kruskal-Wallis test. Immunostaining and western blotting results are compared with Wilcoxon test.

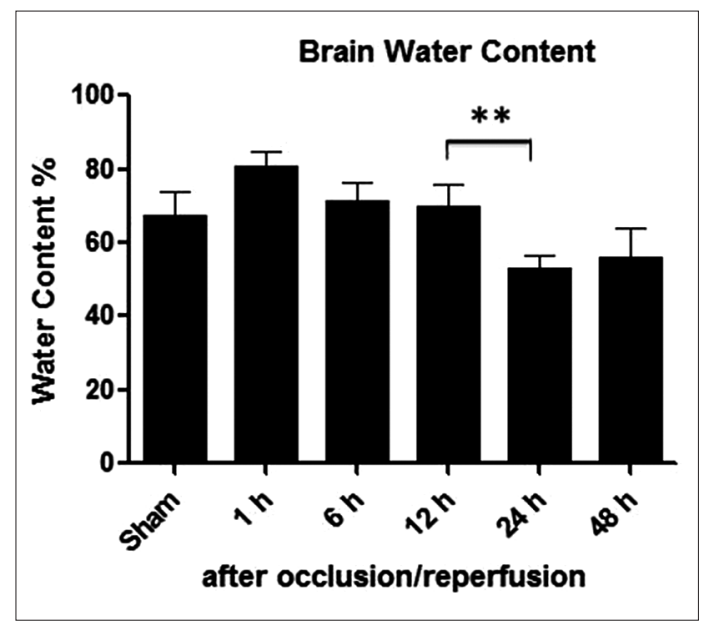

Figure I: Brain water content: Time course change of brain water content was measured in rats at sham, I h, $6 \mathrm{~h}, 12 \mathrm{~h}, 24 \mathrm{~h}$, and $48 \mathrm{~h}$, after four-vessel occlusion/reperfusion (standard error, 4-6 rats/ group $* * P<0.01$ )

\section{RESULTS}

\section{Ischemia/reperfusion}

We used four-vessel global ischemia model in rat in which permanent occlusion of both vertebral arteries was accomplished by bipolar cautery $24 \mathrm{~h}$ before prior to transient bilateral occlusion on the carotid artery using surgical aneurysms clips. Transient bilateral carotid artery occlusion then reduced cerebral blood flow $>95 \%$, which was returned to approximately baseline level after reperfusion.

\section{Brain water content}

Following four-vessel occlusion/reperfusion ipsilateral hemisphere's water content in the ischemic groups increased gradually until $12 \mathrm{~h}$, and subsequently decreased until $48 \mathrm{~h}$ in Figure 1.

\section{Neuronal damage}

Neuronal damage was assessed in brain sections through hippocampus in sham rat and in the ischemic rat at $48 \mathrm{~h}$ after four-vessel occlusion. Figure 2 shows $\mathrm{H}$ and $\mathrm{E}$ and neuronal marker NeuN staining sections at $\times 5$ magnification. Histological evaluation revealed that global cerebral ischemia leads to loss of hippocampal CAl (shown with arrows). In Figure 3, top left $\mathrm{H}$ and $\mathrm{E}$ staining sections at $\times 20$ magnification is shown. CAl neurons appear smaller, shrunken, and dark colored (shown in arrows).

\section{Choroid plexus damage}

Our study considered that not only hippocampal ischemia but also choroid plexus injury. When both vertebral arteries are permanently occluded, and both

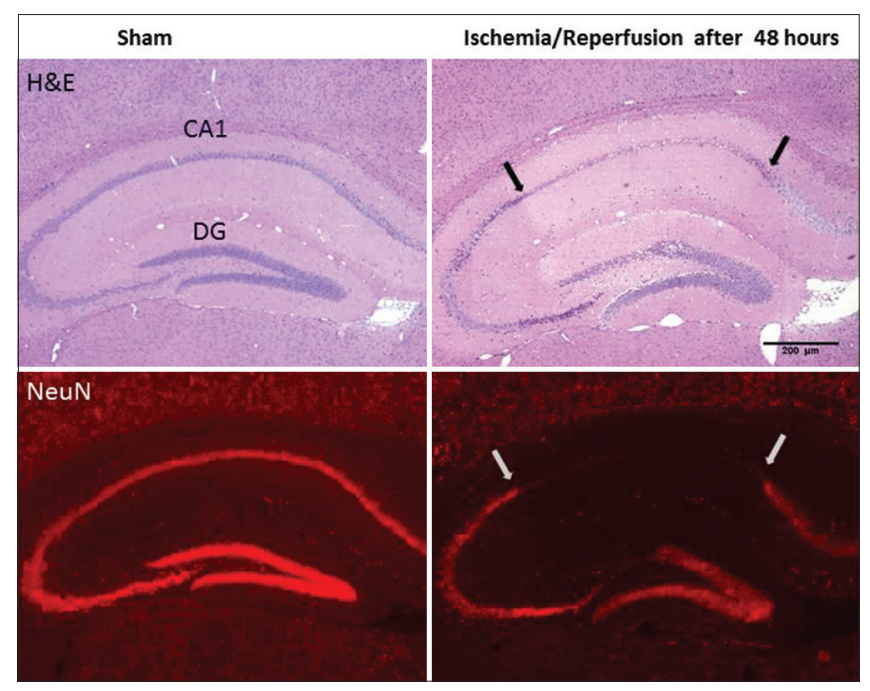

Figure 2: Hematoxylin and eosin and immuno-staining: Histological evaluation with hematoxylin and eosin stained (right top) and NeuN immuno-stained of the hippocampal CAI sector and dentate gyrus in rats (right bottom). Ischemia is not observed in sham group (left) 
carotid arteries are temporarily occluded, there is a drastically reduced blood flow to whole brain areas such as hippocampus and choroid plexuses. Four-vessel ischemia-induced anterior and posterior choroidal artery ischemia in rats caused damage of the choroid plexus tissue. We demonstrated that choroid plexus epithelial cells damaged in Figure 3 (shown by arrows, right side).

\section{Aquaporin 4 and aquaporin 1 expression}

We next evaluated the expression of AQP4 and AQPl after cerebral ischemia/reperfusion injury. The immunostaining results in Figure 4 a show that AQP4

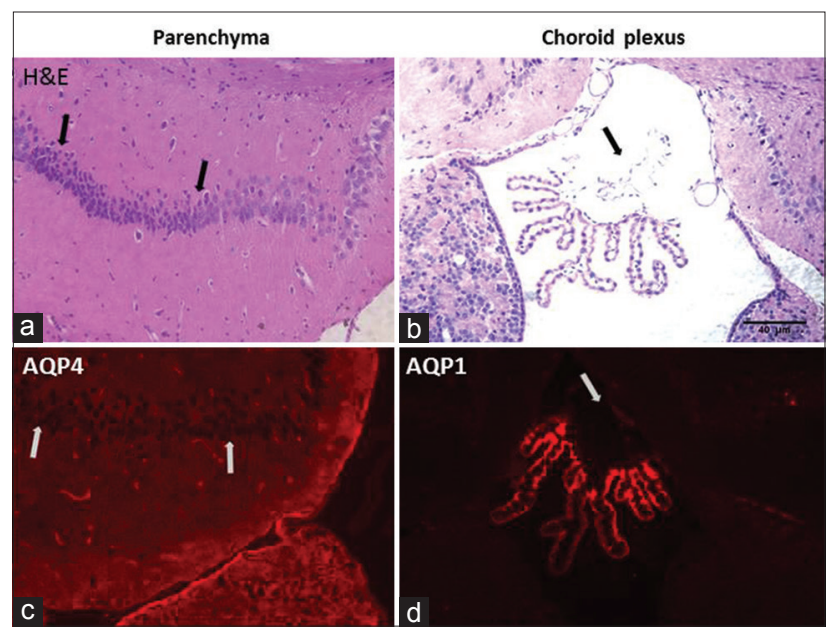

Figure 3: Hematoxylin and eosin staining aquaporins expressions: Histological evaluation with hematoxylin and eosin stained CAI subregion (a) and choroid plexus of the rat at high magnification (b). CAI neurons are observed as small, shrunken and dark pyknotic neurons (shown in arrows). Choroid plexus epithelial cells show damage to apical membrane fragmentation and disruption (arrow).Aquaporins expressions are observed by immune-staining of brain parenchyma and choroid plexus in ischemic rats after 48 h. Aquaporin 4 expression is observed in CAI subregion with stain astrocytes (c).Aquaporin I expression is observed in choroid plexus in third ventricle (d) expression increases after $6 \mathrm{~h}$ but after $12 \mathrm{~h}$ it reduces and later reaches the baseline levels of the sham group although there is not a statistically significant difference in the expression of AQP4 between sham and ischemic groups. AQPl expression shows decrease until $24 \mathrm{~h}$ although change is not statistically significant in Figure 4b. After 24 h, expression levels increase drastically, and the change is statistically significant. In Figure 3, bottom left, we demonstrated that AQP4 expression in CAl subregion and in the right AQPl expression at choroid plexus in the third ventricle.

\section{Western blotting of aquaporin 4}

Quantification of AQP4 expression with western blot showed that change in level is not statistically significant (data not shown).

\section{DISCUSSION}

In this research, we measured AQP4 and AQP1 expression levels in brain and choroid plexus through time course after global cerebral ischemia model. We show that AQP4 expression shows statically not significant changes after ischemic brain edema. On the other hand, AQP1 expression levels showed statistically significant decrease after $24 \mathrm{~h}$ following ischemic brain edema.

Both carotid artery and vertebral arteries supply blood to the whole brain and choroid plexus. Four-vessel occlusion model, which consists of permanent coagulation of vertebral arteries followed by temporary occlusion of carotid arteries after $24 \mathrm{~h}$, give rises to global brain ischemia and choroid plexus ischemia. An extensive neuronal damage in the caudate-putamen and paramedian and CAl zones of the hippocampus is seen after $30 \mathrm{~min}$ of four-vessel occlusion. Pulsinelli et al. noted that forebrain ischemia induced by four-vessel

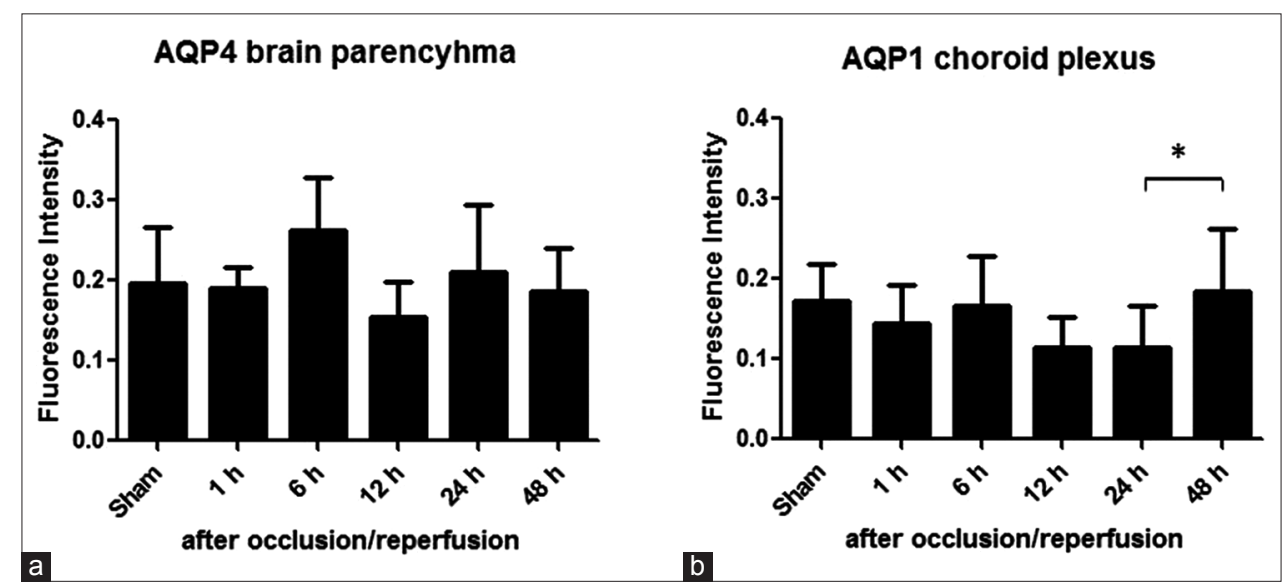

Figure 4: Aquaporins expressions:Time course change of aquaporins expression was evaluated by calculating the ratio of the fluorescence intensity of aquaporins immune-labeling in rat brain parenchyma and choroid plexus in the third ventricle. (a) The aquaporin 4 expression increase at 6 and $24 \mathrm{~h}$ after occlusion/reperfusion was not statistically significant. (b) However, aquaporin I expression increased at $48 \mathrm{~h}$ after occlusion/reperfusion was statistically significant (standard error, 4-6 rats/group $* P<0.05$ ) 
occlusion resulted in choroid plexus necrosis at $6 \mathrm{~h}$ but that choroid plexus morphology returned to normal after 24 h. $^{[5,13]}$

Global ischemia caused by complete interruption of cerebral blood flow results in the rapid breakdown of metabolic and electrophysiological functions of the brain. Associated edema formation has a characteristic time course. ${ }^{[8]}$ First, it causes brain's glucose and oxygen supply to a halt, causing tissues to switch to anaerobic glycolysis. ATP concentration drops below a certain threshold value and cells lose the ability to maintain their membrane potential. Because of the inactivity of the $\mathrm{Na}^{+} / \mathrm{K}^{+}$ATPase, $\mathrm{K}^{+}$leaks out of the cell and leading to an osmotically obliged uptake of water that causes the cells to swell. This phenomenon is known as cytotoxic edema, and it is initiated within minutes after ischemia. Intracellular water accumulation mostly affects astrocytes, and it is believed that astrocyte swelling may have an important role predisposing the brain to further damage. Pericapillary foot processes in astrocytes are the predominant sites of $\mathrm{AQP} 4$ expression in the brain and, therefore, AQP4 is associated with cytotoxic edema. It is previously reported that AQP4 null mice are less prone to this condition as they showed reduced brain water content, infarct size, lesion volume, and lower ICP values while AQP4-overexpressing transgenic mice showed increased cytotoxic edema.

Vasogenic cerebral edema defined as the net extravasation of protein-rich fluid into the brain after the breakdown of the BBB. Following global ischemia, interstitial space shows expansion because of the entrance of serum proteins and fluids from the bloodstream after 6-12 h. ${ }^{[6]}$ The peak of edema is reached after $1-2$ days and tissue swelling can reach to $100 \%$. AQP4 has taught to have a role in fluid clearance from the CNS as it is observed that AQP4 null mice develop more brain edema than wild-type mice. The edema fluid clearance from CNS must involve AQP4-dependent transcellular water flow through astrocyte foot processes into the bloodstream.

Measuring wet-dry ratio of the brain is one of the major methods to quantify brain edema. Our work shows the cytotoxic and vasogenic edema model together with global cerebral ischemia model. Figure 1 shows the time course change of brain water content after different time points following reperfusion. After $\mathrm{l} \mathrm{h}$, an increase in the wet-dry ratio is observed comparing to the sham group. It is thought to be due to the hyperperfusion development after ischemia as a result of disruption cerebral autoregulation. ${ }^{[15]}$ It remains higher until $24 \mathrm{~h}$, decreases after $24 \mathrm{~h}$, and reaches back to the sham levels at $48 \mathrm{~h}$. This result is similar to biphasic edema formation. ${ }^{[15]}$ Cytotoxic edema has occurred during first $6 \mathrm{~h}$. After cerebral autoregulation is obtained, secondary factors increase permeability BBB, and vasogenic edema has formed. The decrease at $24 \mathrm{~h}$ is due to the biphasic nature of cerebral blood flow.

We histologically showed that neural injury in the hippocampal CAl sector and dentate gyrus at $48 \mathrm{~h}$ after 30-min ischemia and reperfusion. Whole pyramidal cells damaged in $\mathrm{CAl}$ subregion and no damage is observed in sham group.

AQP4 expression brain parenchyma and AQPl expression in choroid plexus are measured after occlusion/reperfusion. The increase in AQP4 expression at $6 \mathrm{~h}$ and the following decrease at $12 \mathrm{~h}$ shows that cytotoxic edema is followed by vasogenic edema. A second increase at 24 may indicate the resolution of cytotoxic edema. However, none of these changes were statistically significant. It can be explained by AQP4's bidirectional role in cell membrane during brain edema as it both facilitates cellular water uptake in cytotoxic edema and clearance of extracellular fluid from in vasogenic edema. AQPl plays a part in CSF secretion and absorption. AQPl is expressed in the apical surface of the choroid plexus epithelium. ${ }^{[10]}$ Measurement of AQPl expression levels shows a decrease until $24 \mathrm{~h}$ and statistically significant increase between 24 and $48 \mathrm{~h}$. This decrease can be explained with cell necrosis in choroid plexus and as the cells are replenished and the electrolyte imbalance in choroid plexus is restored, AQPl expression increases.

Histological evaluation of CAl neurons shrinkage in the hippocampal region shows cell necrosis and AQP4 expression in the same region is also shown with immunostaining. This indicates that neuron death. Choroid plexus region also shows cell necrosis and AQPl expression is decreased in the selected region.

Previous studies showed that AQPl and AQP4 expression could be targeted for edema treatment. Our studied showed that AQP4 is continuously active during ischemic edema and its expression in tissues does not change in time course. Since it has a bidirectional role while developing drugs that target AQP4, the time course of edema and type of edema must be considered.

Financial support and sponsorship

Nil.

Conflicts of interest

There are no conflicts of interest.

\section{REFERENCES}

I. Agre P, King LS, Yasui M, Guggino WB, Ottersen OP, Fujiyoshi Y, et al. Aquaporin water channels - From atomic structure to clinical medicine. J Physiol 2002;542(Pt I):3-I6.

2. Badaut J, Lasbennes F, Magistretti PJ, Regli L. Aquaporins in brain: Distribution, physiology, and pathophysiology. J Cereb Blood Flow Metab 2002;22:367-78. 
3. Badaut J. Aquaglyceroporin 9 in brain pathologies. Neuroscience 2010;168:1047-57.

4. Chalela JA, Merino JG, Warach S. Update on stroke. Curr Opin Neurol 2004; 17:447-5I.

5. Dienel GA. Regional accumulation of calcium in postischemic rat brain. J Neurochem 1984;43:913-25.

6. Fujimoto M, Takagi Y, Aoki T, Hayase M, Marumo T, Gomi M, et al. Tissue inhibitor of metalloproteinases protect blood-brain barrier disruption in focal cerebral ischemia. J Cereb Blood Flow Metab 2008;28:1674-85.

7. Klatzo I. Pathophysiological aspects of brain edema. Acta Neuropathol 1987;72:236-9.

8. Marmarou A. A review of progress in understanding the pathophysiology and treatment of brain edema. Neurosurg Focus 2007;22:EI.

9. Nielsen S, Nagelhus EA, Amiry-Moghaddam M, Bourque C, Agre P, Ottersen OP. Specialized membrane domains for water transport in glial cells: High-resolution immunogold cytochemistry of aquaporin-4 in rat brain. J Neurosci 1997;17:171-80.

10. Nielsen S, Smith BL, Christensen El, Agre P. Distribution of the aquaporin CHIP in secretory and resorptive epithelia and capillary endothelia. Proc
Natl Acad Sci U S A 1993;90:7275-9.

II. Oshio K, Watanabe H, Song Y, Verkman AS, Manley GT. Reduced cerebrospinal fluid production and intracranial pressure in mice lacking choroid plexus water channel Aquaporin-I. FASEB J 2005;19:76-8.

12. Papadopoulos MC, Verkman AS. Aquaporin water channels in the nervous system. Nat Rev Neurosci 2013;14:265-77.

13. Pulsinelli WA, Brierley JB, Plum F. Temporal profile of neuronal damage in a model of transient forebrain ischemia. Ann Neurol 1982;1 1:491-8.

14. Pulsinelli WA, Brierley JB. A new model of bilateral hemispheric ischemia in the unanesthetized rat. Stroke 1979;10:267-72.

15. Sandoval KE, Witt KA. Blood-brain barrier tight junction permeability and ischemic stroke. Neurobiol Dis 2008;32:200-19.

16. Simard JM, Kent TA, Chen M, Tarasov KV, Gerzanich V. Brain oedema in focal ischaemia: Molecular pathophysiology and theoretical implications. Lancet Neurol 2007;6:258-68.

17. Thrane AS, Rangroo Thrane V, Nedergaard M. Drowning stars: Reassessing the role of astrocytes in brain edema. Trends Neurosci 20I4;37:620-8.

18. Verkman AS, Anderson MO, Papadopoulos MC. Aquaporins: Important but elusive drug targets. Nat Rev Drug Discov 2014;13:259-77. 